\title{
Inflación colombiana pronosticada con un VAR bayesiano
}

\author{
Norberto Rodríguez Niño* \\ Banco de la República \\ Universidad Nacional de Colombia
}

FECHA DE RECEPCIÓN: MARzo 11 DE 2011

FECHA DE APROBACIÓN: ABRIL 11 DE 2011

\begin{abstract}
Resumen En este trabajo se presentan los resultados de una aplicación de la estimación y pronóstico de modelos de Vectores Autorregresivos usando técnicas bayesianas (BVAR), para la inflación anual colombiana. Se comparan los resultados del uso de diversas especificaciones y de priors; los hiperparámetros de las prior se seleccionan de acuerdo a criterio de bondad del pronóstico. Luego, para las especificaciones seleccionadas, se compara la bondad del pronóstico de la inflación anual generada por los modelos BVAR con el de una caminata aleatoria univariada, y contra los de modelos VAR convencionales. Los resultados muestran que los modelos BVAR mejoran los modelos de los VAR análogos, logrando reducciones de hasta $72.8 \%$ en la Raíz del Error Cuadrático Medio de Pronóstico (RECMP).
\end{abstract}

Abstract This work presents the results of the application of the Vector Autoregressive estimation and prediction by using bayesian techniques (BVAR) for the annual Colombian inflation. The results of the use of diverse specifications and priors are compared. Prior hyperparameters are selected according to the goodness-of-prediction criteria. Then, the goodness-of-prediction of the annual inflation generated by BVAR models is compared to the goodness-of-prediction of a univariated Random Walk for the selected specifications. It is also compared to the conventional VAR models. The results show that BVAR models improve the analog VAR models, reaching deductions of up to $72.8 \%$ in the Prediction Root-Mean-Square Error (RMSE).

Palabras Clave: estimación bayesiana, evaluación de pronóstico, modelos BVAR, inflación, priors.

Keywords: bayesian probability, prediction evaluation, BVAR models, inflation, priors.

\footnotetext{
* Econometrista Asociado, Banco de la República y Profesor Catedrático Titular, Universidad Nacional de Colombia. nrodrini@banrep.gov.co. Agradezco a Cristina Sarmiento por los comentarios, sugerencias y pacientes revisiones de versiones previas y a Eliana González y Andrés González por sus comentarios y sugerencias. Las opiniones aquí expresadas y los errores aún presentes son mi exclusiva responsabilidad y no comprometen la posición oficial de las instituciones con las cuales estoy vinculado.
} 


\section{Introducción}

Actualmente la política monetaria en Colombia, se conduce por medio de un esquema de inflación objetivo (inflation targeting), que tiene como finalidad disminuir las tasas de inflación y buscar la estabilidad del producto alrededor de su tendencia de largo plazo. Bajo esta estructura la toma de decisiones del banco central, reflejadas principalmente en la tasa de interés como instrumento de política, se ve limitada en gran medida a los pronósticos que se hagan sobre la inflación.

El producir adecuados pronósticos de inflación y otros agregados económicos para diferentes horizontes es una tarea difícil. La incertidumbre sobre la verdadera estructura de la economía y los mecanismos de transmisión obligan a los bancos centrales a usar diversas aproximaciones para pronósticar infación en lugar de confiar en un único modelo. Hoy en día existen diversas metodologías para pronosticar variables macroeconómicas como la inflación. Por un lado, el uso de modelos, en especial los multivariados como los Vectores Autorregresivos (VAR), han ganado sostenida popularidad desde que fueron introducidos en el ámbito econométrico hace ya 30 años [22. Por otro lado, los pronósticos por juicio de expertos (combinado o no con modelos estadísticos-econométricos), a pesar de la dificultad de no informar como son usados los datos, son de gran utilidad para bancos centrales, puesto que permiten configurar escenarios factibles de corto plazo que usualmente involucran información no tenida en cuenta por los modelos, además del concenso y las expectativas de los agentes.

En el grupo de modelos multivariantes se pueden ubicar los modelos estructurales grandes, estos modelos tienen la ventaja de producir pronósticos para diversas variables y producir clara intuición económica detras de la dinámica de los pronósticos. El problema de los modelos estructurales grandes es el uso de supuestos restrictivos que deben ser empleados para identificar la estructura de la economía. Por otra parte, los modelos VAR explotan la información presente en las series de tiempo macroeconómicas basados en una cantidad mínima de información estructural de la economía.

Una ventaja de emplear pronósticos basados en modelos es que su bondad puede ser evaluada estadísticamente antes de usar el pronóstico, lo cual no es usualmente posible con los pronósticos de juicio. Las técnicas bayesianas, como es ilustrado en la literatura, pueden ser usadas para cerrar esta brecha entre las dos alternativas, mediante el uso de información preliminar o prior. Los pronósticos no condicionales de la inflación permiten ademas efectuar comparaciones con pronósticos de agentes externos al banco central. Esto posibilita que los directivos juzgen si se presentan diferencias significativas entre la percepción de los niveles de inflación de parte de los agentes del mercado y la suya propia.

La estimación y pronóstico bayesiano se convierte en una alternativa intermedia entre el pronóstico subjetivo y aquel basado en un modelo (VAR por ejemplo) cuya estimación es guiada meramente por los datos. En este trabajo se aborda un ejercicio de pronósticos bayesianos VAR para la economía colombiana. Los resultados de este ejercicio muestran reducciones en la Raíz del Error Cuadrático Medio de Pronóstico (RECMP) relativas a las del VAR frecuentista desde $57.7 \%$ 
en el corto plazo y hasta de $52.8 \%$ en el mediano plazo. Este trabajo pretende ser una contribución empírica a la literatura en este tópico para Colombia.

Este documento esta organizado como sigue: En la sección dos se discuten los indicadores macroeconómicos colombianos; en la sección tres se presentan algunos resultados conceptuales de la estimación bayesiana de modelos VAR. En la sección cuatro se describen los ejercicios de estimación y pronóstico realizados, en la cinco se presentan los resultados de los mismos y finalmente en la sección seis se resume, concluye y recomiendan algunas extensiones al presente trabajo.

\section{El contexto colombiano}

En esta sección brevemente se describe la evolución de los principales indicadores macroeconómicos colombianos durante parte del período de estudio (1984 hasta 2010), la importancia de estos radica en que están dentro del conjunto de información que es usado por el banco central (Banco de la República) para contextualizar los pronósticos y fijar sus metas de inflación.

Durante la década de los noventa, Colombia experimentó una serie de cambios institucionales y de política económica que afectaron el desempeño macroeconómico y posiblemente alteraron la relación entre las variaciones del producto y de los agregados monetarios con la inflación. Dentro de éstos se destacan el proceso de apertura y liberalización comercial y financiera de principios de los años noventa; la reforma constitucional que le dio independencia y autonomía al banco central, estableció explícitamente la defensa del poder adquisitivo de la moneda como el objetivo principal de la política monetaria. A partir de 1992, se anunciaron las metas de inflación y a partir del 2000 se adoptó formalmente un régimen de inflación objetivo. La inflación se redujo de $32.4 \%$ en 1990 a $1.8 \%$ en marzo de 2010, mientras su volatilidad ${ }^{1}$ se redujo de 1.7 a 1.2 , respectivamente, llegando a su máximo de 3.7 a mediados de 1999. Recientemente la volatilidad ha mostrado incrementos presentando nuevamente niveles de hasta 1.9 en el 2009 y reducciones hasta llegar a 0.2 a finales de 2010 .

La inflación colombiana aunque continúa en niveles bajos, comparada con la de sus principales socios comerciales, es aún objeto de preocupación para las autoridades económicas y para el público en general. La inflación, entendida como la tasa de variación del nivel observado de precios, los cuales se espera presenten niveles de equilibrio, hace considerar la estimación de modelos multivariantes que contemplen la posible existencia de dichos equilibrios de largo plazo. En este trabajo se modela la presencia de cointegración tanto con la alternativa de Johansen como con la bayesiana tradicional.

Es de anotar que resultados de otros trabajos de evaluación de pronósticos de la inflación colombiana, a diferentes horizontes y diferentes periodicidades, muestran señales variadas. Los modelos utilizados actualmente por el banco central generan valores de error Cuadrático Medio de Predicción (RECMP o FMSE) de la inflación anual, para un año adelante, entre 1,5 y 9,7. Por ejemplo, 9 .

\footnotetext{
${ }^{1}$ Medida como la desviación estándar móvil de las doce inflaciones anuales más
} recientes. 
presenta RECMP de $4.4 \%$ a un trimestre y de $9.7 \%$ a un año en una especificación, mientras que encuentran de $7.8 \%$ a $16.5 \%$, a los mismos horizontes, usando otra especificación. [15] reporta $4.4 \%$ a un trimestre y $1.6 \%$ a un año (usando como período de evaluación enero 2004 hasta enero 2006). 8 reporta $1.7 \%$ a $2.6 \%$ usando datos trimestrales del período 2001: I - 2005: IV. [10], usando modelos factoriales dinámicos y series mensuales logra reducciones de $70 \%$ en RECMP relativo a un modelo AR univariante, el período de evaluación es enero 2005 a junio de 2008.

\section{Vectores autorregresivos bayesianos}

Esta sección se ocupa de los resultados conceptuales de estimación y pronósticos bayesianos en modelos VAR. Primero, por ser punto de referencia, se presenta la notación de los VAR frecuentistas y luego los conceptos de VAR bayesiano.

\subsection{Modelamiento VAR Frecuentista}

Considere el modelo $\operatorname{VAR}(\mathrm{p})$

$$
y_{t}=\delta+\Phi_{1} y_{t-1}+\ldots+\Phi_{p} y_{t-p}+\varepsilon_{t}
$$

ó

$$
\mathbf{y}=\left(X \otimes I_{k}\right) \beta+\varepsilon
$$

con

$$
\varepsilon_{t} \sim N\left(0, \Sigma_{\varepsilon}\right)
$$

donde $y_{t}$ denota un vector columna de $k$ variables incluidas en el VAR para el período $t, t=1,2, \cdots, T ; \mathbf{y}=\operatorname{vec}\left(y_{1}, y_{2}, \ldots, y_{T}\right)$, vector de $k p \times 1 ; X=$ $\left[X_{0}^{\prime}, X_{1}^{\prime}, \ldots, X_{T-1}^{\prime}\right]^{\prime}$, con $X_{t}=\left[\begin{array}{lll}1 & y_{t}^{\prime} & y_{t-1}^{\prime}\end{array} \ldots y_{t-p+1}^{\prime}\right] ; \otimes$ el producto Kronecker; el vector columna $\varepsilon_{t}$ de dimensión $k$ denota el termino de error, el cual cuantifica el punto hasta donde $y_{t}$ puede ser determinado exactamente por una combinación lineal de valores pasados de $y_{t}$, con ponderaciones dadas por las constantes contenidas el el vector $\delta$ y en las matrices $\Phi_{i} ; \beta$ representa al vector de $k+k^{2} p$ parámetros generado por el operador $\operatorname{vec}\left(\delta, \Phi_{1}, \ldots, \Phi_{p}\right)$, así por ejemplo para un VAR bivariado, $k=2$, con $p=2$ rezagos se tiene,

$$
\begin{aligned}
& y_{1 t}=\delta_{1}+\phi_{1,1}^{1} y_{1, t-1}+\phi_{1,2}^{1} y_{2, t-1}+\phi_{1,1}^{2} y_{1, t-2}+\phi_{1,2}^{2} y_{2, t-2}+\varepsilon_{1 t} \\
& y_{2 t}=\delta_{2}+\phi_{2,1}^{1} y_{1, t-1}+\phi_{2,2}^{1} y_{2, t-1}+\phi_{2,1}^{2} y_{1, t-2}+\phi_{2,2}^{2} y_{2, t-2}+\varepsilon_{2 t}
\end{aligned}
$$

con

$$
\beta=\left[\begin{array}{lllll}
\delta_{1} \delta_{2} & \phi_{1,1}^{1} \phi_{2,1}^{1} \phi_{1,2}^{1} \phi_{2,2}^{1} \phi_{1,1}^{2} \phi_{2,1}^{2} \phi_{1,2}^{2} \phi_{2,2}^{2}
\end{array}\right]^{\prime}
$$

Otra notación útil en otros contextos, se puede encontrar en [1, sección 10.1]. 
La función de verosimilitud para el proceso gaussiano resulta entonces en,

$$
\begin{aligned}
& l(\beta \mid y)=\left(\frac{1}{2 \pi}\right)^{k T / 2}\left|I_{T} \otimes \Sigma_{\varepsilon}\right|^{-1 / 2} \times \\
& \exp \left[-\frac{1}{2}\left(\left(y-\left(X \otimes I_{k}\right) \beta\right)^{\prime}\left(I_{T} \otimes \Sigma_{\varepsilon}^{-1}\right)\left(y-\left(X \otimes I_{k}\right) \beta\right)\right]\right.
\end{aligned}
$$

Se ha mostrado [18, que el estimador máximo verosímil de $\beta$, bajo el supuesto de que $\varepsilon_{t}$ se distribuye normal multivariante $\left(0, \Sigma_{\varepsilon}\right)$, en cuyo caso coincide con el de mínimos cuadrados, esta dado por,

$$
\hat{\beta}=\left[\left(X^{\prime} X \otimes \Sigma_{\varepsilon}^{-1}\right)\right]^{-1}\left[\left(X^{\prime} \otimes \Sigma_{\varepsilon}^{-1}\right) y\right]
$$

y la matriz de varianza-covarianza está dada por,

$$
\Sigma_{\hat{\beta}}=\left[\left(X^{\prime} X \otimes \Sigma_{\varepsilon}^{-1}\right)\right]^{-1}
$$

Resultados básicos sobre modelos VAR convencionales o frecuentistas, se pueden encontrar, por ejemplo, en Judge [13] y más completos en [18] el cual contiene, además, una revisión sobre VAR bayesiano.

La estructura de los VAR convencionales conlleva un inconveniente serio. En la práctica es frecuente encontrar modelos VAR que estimados sin restricciones ${ }^{2}$ requiere estimar un gran número de parámetros, aún más en el caso de una cantidad moderada de variables y rezagos. Esto usualmente produce errores estándar grandes de los coeficientes estimados, lo cual puede deberse a que los coeficientes son en efecto cero o que los datos pueden no ser lo suficientemente informativos para producir estimaciones precisas de los coeficientes.

\subsection{Modelamiento VAR bayesiano}

Una solución alternativa al problema de sobre-parametrización es la aproximación bayesiana. El problema de "sobre-parametrización" frecuentemente resulta en buen ajuste dentro de muestra pero un pobre desempeño del pronóstico fuera de muestra. Por esta razón, en este trabajo se emplean técnicas bayesianas, como las propuestas [164, en las cuales a priori se imponen restricciones inexactas sobre las matrices de coeficientes, con lo cual se configura un método útil para reducir la dimensionalidad asociada con el VAR no restringido, lo cual ha mostrado generar pronósticos más adecuados.

La idea detras del procedimiento VAR bayesiano es asignar menos peso a los rezagos mas distantes pero sin restringirlos a cero, en tanto que permiten que este supuesto sea sobreajustado si se tiene evidencia fuerte en los datos que indique otra cosa. En la práctica, esto es implementado especificando distribuciones prior para los coeficientes del VAR, los cuales son tratados como variables aleatorias con valores esperados especificados previamente (a priori), y con la concentración

\footnotetext{
${ }^{2}$ Es decir, todos los coeficientes en todas las $k$ ecuaciones son estimados libremente.
} 
(tightness) de las distribuciones las cuales son determinadas por un conjunto de hiperparámetros. Esta información es luego incorporada en la estimación junto con la información muestral para obtener la estimación final o posterior.

Los modelos VAR bayesianos pueden ser vistos como un intermedio (puente) entre modelos multivariados de series temporales usados sólo para generar pronóstico y modelos interpretables de ecuaciones simultáneas. En el mismo sentido que la estimación de modelos de Equilibrio General Dinámicos Estocásticos (DSGE, por sus iniciales en inglés) el modelo bayesiano es visto como un intermedio entre calibración y estimación pura.

En el contexto bayesiano tradicional, el vector de parámetros $\beta$ sigue $a$ priori una distribución normal multivariada con media conocida $\beta^{*}$ y matriz de varianza-covarianza $V_{\beta}$, consecuentemente la densidad prior es escrita como

$$
f(\beta)=\left(\frac{1}{2 \pi}\right)^{k^{2} p / 2}\left|V_{\beta}\right|^{-1 / 2} \exp \left[-\frac{1}{2}\left(\beta-\beta^{*}\right) V_{\beta}^{-1}\left(\beta-\beta^{*}\right)\right]
$$

En esta aplicación se sigue a [4] al especificar la desviación estándar de la distribución prior para el rezago $l$ de la variable $j$ en la ecuación $i$, es decir, el elemento $i, j$ de la matriz $l$ de coeficientes $\Phi_{l}$, denotado por $S(i, j, l)$ acorde a la siguiente representación.

Combinando (10) con la verosimilitud (7), se obtiene la densidad posterior ${ }^{3}$ obteniendo el resultado deseable y facilitador de conjugamiento (véase [13]), es decir, normalidad, densidad dada por

$$
f(\beta \mid y) \propto \exp \left[-\frac{1}{2}(\beta-\bar{\beta})^{\prime} \bar{\Sigma}_{\beta}^{-1}(\beta-\bar{\beta})\right]
$$

donde la media (y moda) posterior es 4

$$
\bar{\beta}=\left[V_{\beta}^{-1}+\left(X^{\prime} X \otimes \Sigma_{\varepsilon}^{-1}\right)\right]^{-1}\left[V_{\beta}^{-1} \beta^{*}+\left(X^{\prime} \otimes \Sigma_{\varepsilon}^{-1}\right) y\right]
$$

y la matriz de varianza-covarianzas posterior es

$$
\bar{\Sigma}_{\beta}=\left[V_{\beta}^{-1}+\left(X^{\prime} X \otimes \Sigma_{\varepsilon}^{-1}\right)\right]^{-1}
$$

ó

$$
\bar{\Sigma}_{\beta}^{-1}=V_{\beta}^{-1}+\Sigma_{\hat{\beta}}^{-1}
$$

lo anterior implica que tanto la estimación puntual como la de la matriz de precisión (inversa de la matriz de varianzas-covarianzas) se pueden interpretar como promedio ponderado de la media y precisión a priori respectivas y las estimaciones frecuentistas; además, se mantiene el ya tradicional resultado de que ante mayor incertidumbre en las priors (varianzas prior tendiendo a infinito)

${ }^{3}$ Excepto por una constante de proporcionalidad.

${ }^{4}$ Otra representación que no requiere invertir $V_{\beta}$ se encuentra en [18, sec. 5.4.2], la cual es útil cuando no se tiene información precisa sobre algunos de los coeficientes. 
mayor similitud entre la estimación bayesiana y la estimación frecuentista, y, en el otro extremo a mayor certidumbre en las priors (varianzas a priori pequeñas) las densidades posterior estarán mas cerca de las priors. Por simplicidad computacional, en este trabajo se supone que $\Sigma_{\varepsilon}$ es fija y conocida ${ }^{5}$.

La media posterior así obtenida y presentada en $(12)$ tiene la misma forma del estimador de Theil o de Cresta (Ridge Regression) usado en modelos lineales cuando los coeficientes son estimados con restricciones estocásticas, con lo cual se resuelve el problema de multi-colinealidad presente en los VAR no restringidos. Otra interpretación posible, y a veces útil, es el uso de información dummy explicada como información adicionada al sistema de ecuaciones del VAR. Véase [1, sección 10.2].

En la práctica, la media prior $\beta^{*}$ y la varianza prior $V_{\beta}$ necesitan ser especificadas. Si todos los parámetros son considerados concentrados (shrinking) hacia cero, la media prior nula puede ser especificada. De acuerdo con Litterman [17], la varianza prior puede ser dada por

$$
v_{i j}(l)= \begin{cases}(\lambda / l)^{2} & \text { si } i=j \\ \left(\lambda \theta \sigma_{i, i} / l \sigma_{j, j}\right)^{2} & \text { si } i \neq j\end{cases}
$$

donde $v_{i j}(l)$ es la varianza prior del $(i, j)$-ésimo elemento de $\Phi_{l}, \lambda$ resulta ser la desviación estándar prior de los elementos de la diagonal de la matriz de primeros rezagos, $\Phi_{1}$ en (1); $\theta$ es una constante en el intervalo $(0,1)$, que controla la incertidumbre sobre los parámetros de primer rezago.

Para rezagos propios de orden mayor a uno, la incertidumbre disminuye a una velocidad dada por $\frac{\lambda}{l}$. $\sigma_{i, i}^{2}$ es el elemento $i$-ésimo de la diagonal de $\Sigma_{\varepsilon}$. Los términos determinísticos tienen varianza prior difusa. En la práctica, se reemplaza $\sigma_{i, i}^{2}$ por el elemento $i$-ésimo de la diagonal del estimador Máximo Verosímil de $\Sigma_{\varepsilon}$ en el modelo no restringido. El hiper-parámetro $\lambda$ controla la concentración alrededor de cero de la distribución del primer rezago de la variable dependiente 6

Por ejemplo, para un modelo $\operatorname{BVAR}(2)$ bivariado,

$$
\begin{aligned}
& y_{1 t}=0+\phi_{1,1}^{1} y_{1, t-1}+\phi_{1,2}^{1} y_{2, t-1}+\phi_{1,1}^{2} y_{1, t-2}+\phi_{1,2}^{2} y_{2, t-2}+\varepsilon 1 t \\
& y_{2 t}=0+\phi_{2,1}^{1} y_{1, t-1}+\phi_{2,2}^{1} y_{2, t-1}+\phi_{2,1}^{2} y_{1, t-2}+\phi_{2,2}^{2} y_{2, t-2}+\varepsilon_{2 t}
\end{aligned}
$$

\footnotetext{
${ }^{5}$ Para estudiar otros casos se puede consultar [1, sección 10.2]

${ }^{6}$ Es posible configurar valores de $\lambda$ diferentes para cada ecuación.
} 
con matriz de varianza - covarianza prior

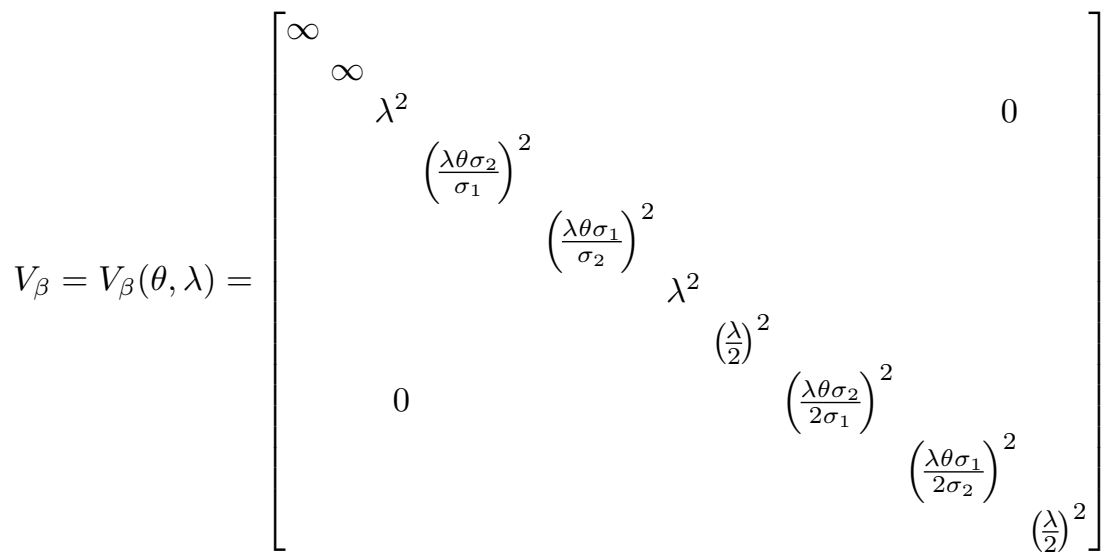

Los valores de cero para los interceptos de las ecuaciones se usan por generalizar, con el supuesto de incertidumbre total a priori sobre dichos valores. Note que si, por ejemplo, $\theta=0, V_{\beta}$ se reduce a:

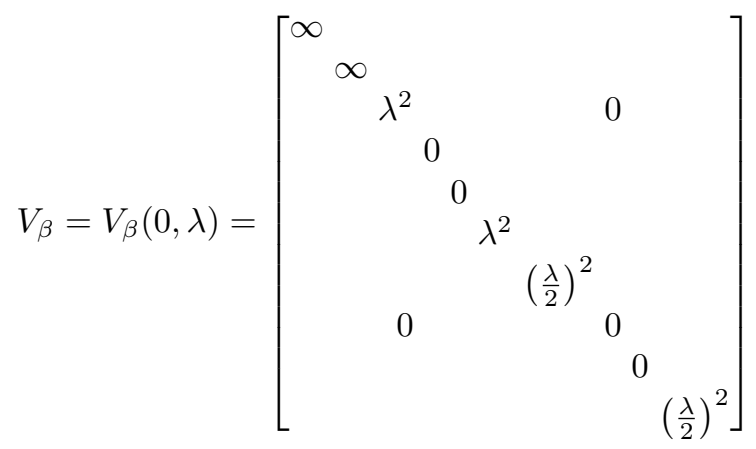

Puesto que $\theta(\operatorname{con} 0<\theta \leq 1)$ controla todas las varianzas de los parámetros endógenos, es llamado parámetro de precisión total, mientras que $\lambda$ es denominado como el parámetro de decaimiento (decay parameter). Note que la varianza de los coeficientes de una misma ecuación de un rezago es menor a medida que el rezago es mas grande 7 La figura 1 muestra esquemáticamente los resultados anteriores usando cuatro rezagos, e hiperparámetors arbitrarios $\lambda=0.6$ y $\theta=0.1$. Valores distintos de estos últimos generan gráficas análogas, con debidos cambios en escala.

Para la estimación bayesiana de sistemas integrados, la media prior es igualada a uno para el primer rezago de cada variable en su propia ecuación y todos los otros coeficientes igualados a cero. Por ejemplo, para un modelo BVAR(2) bivariado,

$\overline{7}$ Valores de $\lambda$ muy grandes junto con $\theta \neq 0$, generan estimaciones puntuales cercanas a las frecuentistas. 

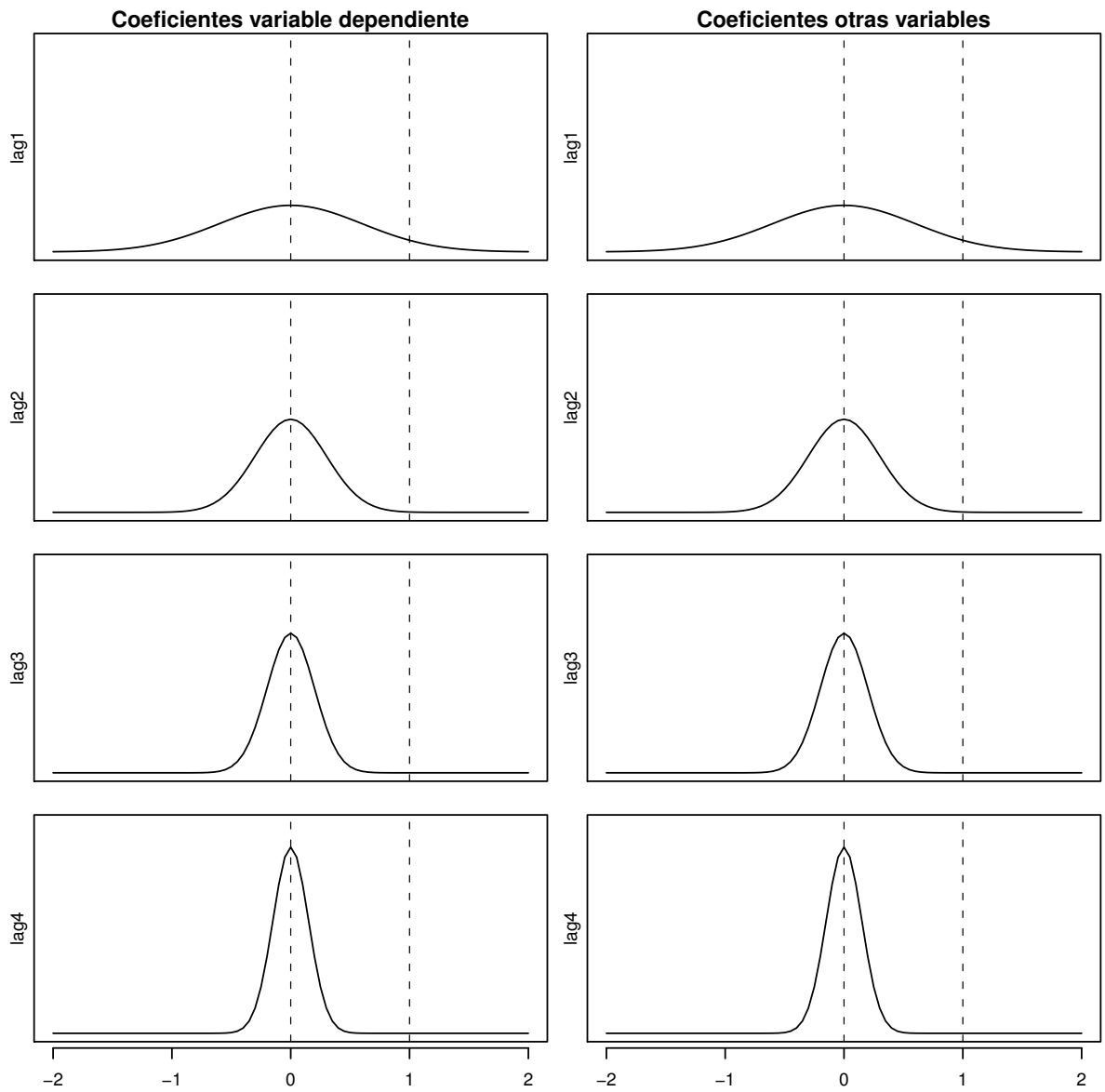

Figura 1. Estructura de las densidades prior de Litterman no cointegración.

las prior serían,

$$
\begin{aligned}
& y_{1 t}=0+1 y_{1, t-1}+0 y_{2, t-1}+0 y_{1, t-2}+0 y_{2, t-2}+\varepsilon_{1 t} \\
& y_{2 t}=0+0 y_{1, t-1}+1 y_{2, t-1}+0 y_{1, t-2}+0 y_{2, t-2}+\varepsilon_{2 t}
\end{aligned}
$$

es decir

$$
\begin{aligned}
& y_{1 t}=y_{1, t-1}+\varepsilon_{1 t} \\
& y_{2 t}=y_{2, t-1}+\varepsilon_{2 t}
\end{aligned}
$$

esto es, cada variable es una caminata aleatoria, pero no necesariamente independientes pues $\operatorname{Cov}\left(\varepsilon_{1 t}, \varepsilon_{2 t}\right)$ no se restringe a ser cero. Este supuesto es razonable pues las caminatas aleatorias usualmente generan pronósticos adecuados para 
series de tiempo macro-económicas, sin embargo, esta condición es considerada solamente a priori, pues la densidad posterior depende de los datos. La figura 2 muestra este esquema, usando los mismos cuatro rezagos y valores de hiperparámetros que antes. Note que la única diferencia es la ubicación de la densidad prior del primer rezago de la primera variables, antes centrada en cero y ahora en uno. En esta especificación tradicional no se imponen las restricciones de Johansen $12]^{8}$
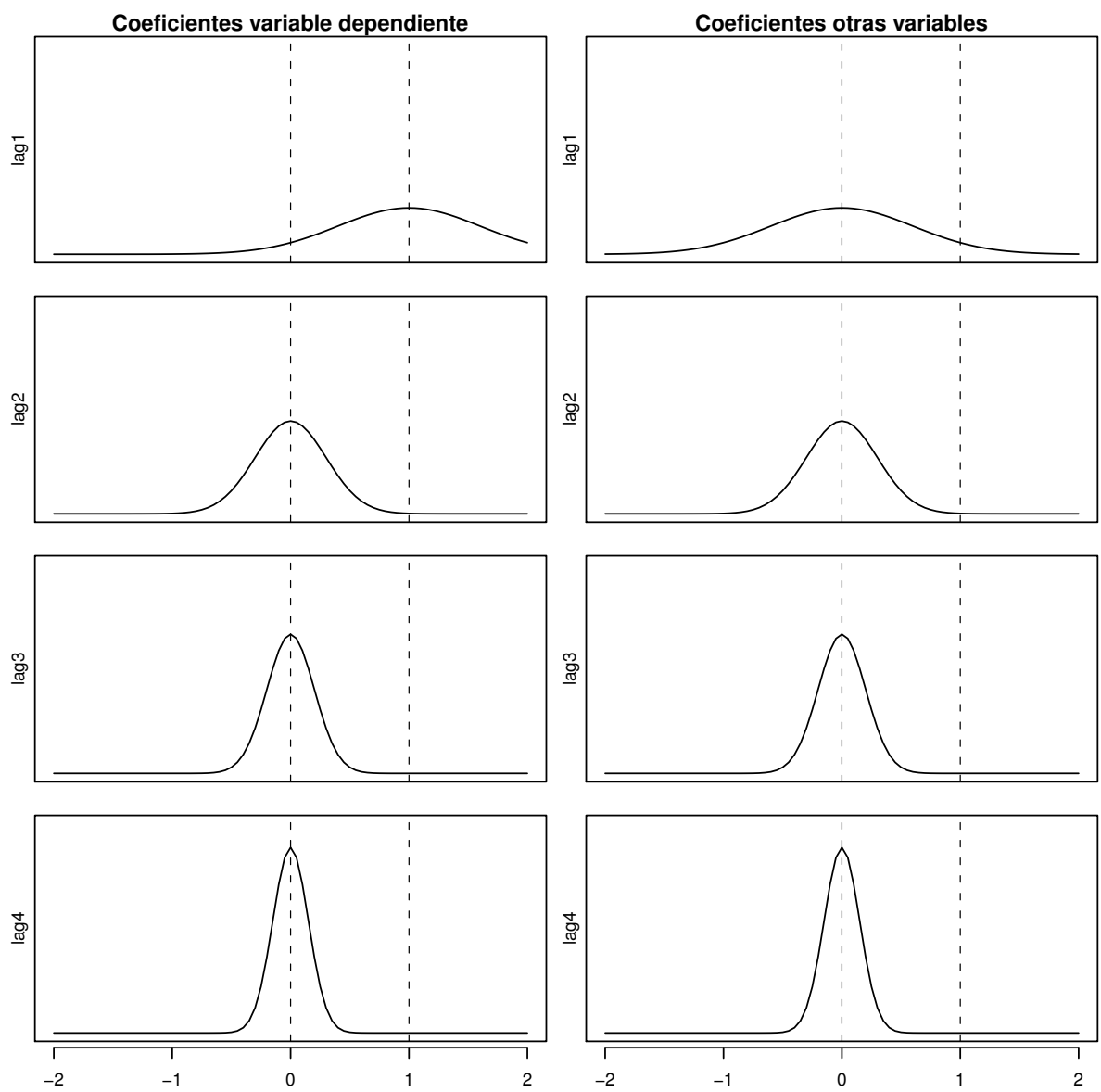

Figura 2. Estructura de las densidades prior de Litterman con cointegración.

\footnotetext{
${ }^{8}$ Una revisión de literatura de BVAR con restricciones de cointegración está en [14].
} 


\subsection{Pronósticos en modelos BVAR}

Los pronósticos y los Impulsos-Respuesta (IR), se calculan de forma convencional y tiene la propiedad de ser consistentes si $T \rightarrow \infty$. Pero en la práctica el investigador debe trabajar con un $T$ finito, además, obtener intervalos de credibilidad en forma analítica es difícil, en consecuencia en la práctica se recurre a métodos de simulación.

El procedimiento bootstrap es utilizado para estimar errores estándar del pronóstico [17/5]. Se generan $B$ simulaciones y en cada una de estas se realizan los siguientes pasos:

- El procedimiento genera con reemplazamiento, una muestra de tamaño igual a la cantidad de observaciones disponibles, $T$, y números enteros aleatorios con distribución uniforme, $I_{t}$, donde $t=1, \ldots T$.

- Una nueva observación, $\hat{y}_{t}$, es obtenida como la suma de los pronósticos basados en los coeficientes estimados, más el vector de residuos de la muestra, $I_{t}$; esto es,

$$
\hat{y}_{t}=\sum_{j=1}^{p} \hat{\Phi}_{j} y_{t-j}+\hat{\varepsilon}_{I_{t}}
$$

los valores iniciales $y_{-p+1}, \cdots, y_{-1}, y_{0}$ son retenidos como fijos.

- Un nuevo modelo BVAR es estimado usando las pseudo-observaciones, así obtenidas, recurrentemente se obtiene un valor de predicción usando las observaciones mas recientes:

$$
\hat{y}_{T+l \mid T}=\hat{\delta}+\sum_{j=1}^{p} \hat{\Phi}_{j} y_{T+l-j} .
$$

- La medida de Error Cuadrado Medio del Pronóstico, ECMP, l-pasos adelante es

$$
\operatorname{ECMP}(l)=\frac{1}{B} \sum_{i=1}^{B}\left(\hat{y}_{T+l \mid T}^{i}-\overline{\hat{y}}_{T}\right)^{2}
$$

donde $\overline{\hat{y}}_{T}=(1 / B) \sum_{i=1}^{B} \overline{\hat{y}}_{T}^{i}$.

\subsection{Medidas de evaluación de pronóstico}

La experiencia en la aplicación y uso de modelos BVAR ha mostrado que la mejor elección de los hiper-parámetros $\left(\beta\right.$ y $\left.V_{\beta}\right)$ se obtiene de la evaluación de pronósticos fuera de muestra; al respecto véase [23]; esa es la alternativa seguida en este trabajd 9 Con esa idea como objetivo, la estrategia de evaluación sigue los lineamientos del conocido Rolling de ventanas crecientes (expanding windows), el cual es un procedimiento en el que se generan pronósticos secuencialmente.

\footnotetext{
${ }^{9}$ Una alternativa es usar métodos bayesianos jerárquicos, los cuales emplean densidades prior sobre los hiperparámetros, véase [1].
} 
El primer período de datos para la estimación se extiende desde el primer trimestre de 1984 hasta el último trimestre de 1999, así ningún modelo es estimado con menos de 60 observaciones. Los coeficientes estimados en cada modelo VAR y BVAR son usados para generar pronósticos fuera de la muestra para horizontes $h=1,2, \ldots, 12$ trimestres adelante, es decir, para 2000-I en adelante. Hecho esto, la muestra es ampliada con las observaciones del siguiente período, primer trimestre de 2000, cada modelo es re-estimado y esos coeficientes re-estimados son empleados para producir el pronóstico del período que inicia en 2000-II. Este procedimiento es continuado hasta el final del ciclo del que se dispone sobre observaciones, en este caso hasta incluir las observaciones de 2009-IV y solo el pronóstico de un paso adelante es considerado.

Las predicciones del IPC y PIB son registradas y transformadas a variaciones año a año, i.e. inflación anual y crecimiento anual del PIB; las prediciones de la tasa de interés no son transformadas. Finalmente, las predicciones de las variables en escalas originales son comparadas con los valores observados en diferentes trimestres hacia adelante y los errores de predicción son calculados y almacenados.

Cinco estadísticas se usan para medir la capacidad de pronóstico individual fuera de muestra, el Error Medio de Pronóstico (EMP), el Error Absoluto Porcentual Medio de Pronóstico (EAPMP), la Raíz del Error Cuadrático Medio de Pronóstico (RECMP), la Raíz del Error Cuadrático Porcentual Medio de Pronóstico(RECPMP) y la estadística $U$ de Theil. Sus fórmulas aparecen a continuación:

$$
\begin{gathered}
E M P(h)=(p-h+1)^{-1} \sum_{r=0}^{p-l}\left(Z_{T-p+r+h}-\hat{Z}_{T-p+r+h}\right) \\
\operatorname{EAPMP}(h)=(p-h+1)^{-1} \sum_{r=0}^{p-h} \frac{\left|\hat{Z}_{T-p+r+h}-Z_{T-p+r+h}\right|}{\left|Z_{T-p+r+h}\right|} * 100 \\
\operatorname{RECMP}(h)=\sqrt{(p-h+1)^{-1} \sum_{r=0}^{p-h}\left(\hat{Z}_{T-p+r+h}-Z_{T-p+r+h}\right)^{2}} \\
R E C P M P(h)=\sqrt{(p-h+1)^{-1} \sum_{r=0}^{p-h}\left(\frac{\hat{Z}_{T-p+r+h}-Z_{T-p+r+h}}{Z_{T-p+r+h}} * 100\right)^{2}} \\
U-T h e i l(h)=\frac{\sum_{r=0}^{p-h}\left(\hat{Z}_{T-p+r+h}-Z_{T-p+r+h}\right)^{2}}{\sum_{r=0}^{p-h}\left(Z_{T-p+r+h}-Z_{T-p+r}\right)^{2}}
\end{gathered}
$$

donde $h=1,2,3, \ldots$, denota el horizonte de tiempo (número de trimestres) sobre el cual se realiza el pronóstico, $T$ es el tamaño de la serie efectiva usada par realizar la predicción, $p$ indica el número de observaciones que son excluidas al final de la serie para hacer comparaciones fuera de muestra, $r=1, \ldots, p$ indica el aumento de tamaño de la sub-muestra usada para la evaluación. 
Note que la $U$ - Theil compara cada modelo a cada horizonte contra el pronóstico obtenido de un modelo de Caminata Aleatoria Univariada.

Adicionalmente, se calcula la estadística de Diebold y Mariano [3], DM en adelante bajo este enfoque, se parte de la hipótesis nula en la cual el nuevo modelo no genera mejores pronósticos que el existente o de referencia, contra la alternativa que el modelo en consideración mejora al modelo de referencia (benchmark). Interesa por tanto rechazar la hipótesis nula.

$$
\begin{aligned}
& H_{0}=D M_{i} \geq 0 \\
& H_{a}=D M_{i}<0
\end{aligned}
$$

para $i=1$ y 2 , usando

$$
\begin{gathered}
D M_{1}=(O-P N)^{2}-(O-P R)^{2} \\
D M_{2}=|O-P N|-|O-P R|
\end{gathered}
$$

donde $O$ : Dato observado, $P R$ : Pronóstico modelo referencia, $P N$ : Pronóstico modelo nuevo, obs : número de observaciones usadas para pronosticar.

Bajo la hipótesis nula, tanto $D M_{1}$ como $D M_{2}$ se distribuyen cada una como una normal estándar. Así, lo deseable es obtener valores para estas estadísticas menores que cero (0) con $p$-valores pequeños.

Adicionalmente, se usa la corrección propuesta por Harvey et al (1997), la cual presenta una aproximación más potente cuando se aplica en muestras pequeñas, tiene en cuenta la posible correlación serial de los errores de pronóstico, y emplea aproximación a la densidad t-student, en lugar de la Normal.

\section{Metodología}

En esta sección se presenta primero una breve descripción de la base de datos y la metodología empleada, y luego se resumen los principales resultados de los ejercicios realizados.

La base de datos tiene periodicidad trimestral desde 1984-I hasta 2010-IV. Contiene el Producto Interno Bruto a precios de 1994, PIB; el índice de Precios al Consumidor, IPC, calculado como promedio geométrico de los datos mensuales, este último estacionalmente ajustado y con base 1994; la Tasa de Interés de depósitos a término fijo 90 días, (calculada por el Banco de la República); la Base monetaria, en este caso el dato de cada trimestre corresponde al promedio aritmético de los datos mensuales (fin de período) fuente base de datos semanal del Banco de la República; el índice de la tasa de cambio real efectiva es calculado con el IPP de ocho países socios comerciales de Colombia y tiene fuente SGEE del Banco de la República, el cual se refiere al promedio que incluye 8 paises socios comerciales y los respectivos índices de precios del productor.

Todas las variables, excepto las tasa de interés, están expresadas en logaritmos, se desestacionalizan con el procedimiento TRAMO-SEATS (véase [7]). Ningún 
de los modelos considerados incluye variables dicotómicas ni estacionales, ni de otra clase, tampoco tendencias deterministas.

Se estiman diferentes modelos VAR. En cuanto al ordenamiento de las series, se siguen los lineamientos de Eichenbaum y Evans [6], así como Christiano, Eichenbaum y Evans [2. Los modelos VAR se estiman con las siguientes variables: IPC, PIB, BASE, Tasa de Interés de los CDT 90 días y la Tasa de Cambio Nominal, con ese ordenamiento de la variable mas endógena a la mas exógena, ordenamiento que según resultados de Eichenbaum y Evans [6] evita el "acertijo de los precios" (price puzzle). Es decir, puede evitar el resultado de otros modelos VAR, donde los precios se incrementarían significativamente luego de un alza en la tasa de interés.

Las alternativas de especificación son las siguientes: para la primera, se usan todas las series diferenciadas, en la segunda en niveles y sin considerar restricciones de cointegración (rango de cointegración $r=0$ ) y la tercera y última, series en niveles y considerando como restricción una relación de cointegración $(r=1)$.

\section{Resultados principales}

Para la elección de los hiper-parámetros $\lambda$ y $\theta$, se evalua cuál combinación de valores de estas constantes produce mejores pronósticos de la inflación anual, según estadística de RECMP y usando el período 2000-2006. Luego de realizar una búsqueda de malla medianamente exhaustiva, según longitud de rezago e hiper-parámetros, los resultados de bondad del pronóstico son alentadores. Los valores de $\lambda$ y $\theta$, encontrados para cada modelo, son los que se muestran en el cuadro 1, los resultados que siguen utilizan estas especificaciones:

\begin{tabular}{cccccc}
\hline \hline Especificación Rango Co-integr. rezago & $\lambda$ & $\theta$ & RECMP \\
\hline Diferencias & 0 & 3 & 0.01 & 0.01 & 2.043 \\
Niveles & 0 & 1 & 0.01 & 0.01 & 0.762 \\
Niveles & 1 & 4 & 0.90 & 0.90 & 2.518 \\
\hline \hline
\end{tabular}

Fuente: Cálculos del autor

Cuadro 1. Especificación e hiperparámetros para los diferentes BVAR.

Se ha escogido iniciar el período de pronóstico fuera de la muestra tipo rolling en 2000-I, para disponer de suficientes datos que consideren el cambio de régimen posterior a la recesión de 1998-1999. Sin embargo, ejercicios con submuestras de este período muestran resultados similares a los reportados, y están disponibles del autor a solicitud del interesado.

Al considerar los resultados de bondad del pronóstico de los modelos BVAR (no presentados por brevedad) es claro que, de no seleccionar adecuadamente los valores de los hiperparámetros, se puede estar sujeto a errores de pronóstico 
bastante grandes, generando incluso pronósticos más imprecisos que los obtenidos al usar modelos VAR equivalentes. Pero por otro lado, la adecuada selección de dichas constantes es de gran ayuda, como se describe más adelante.

El cuadro 2 presenta los resultados de evaluación de la bondad de los pronósticos de la inflación anual para el período 2005-I hasta 2010-IV. En adelante y por brevedad, solo se presentan las medidas de bondad de pronóstico para horizontes 1, 2, 4, 6 y 8 trimestres en adelante, así como el promedio de 1 a 12 trimestres ${ }^{10}$. Como es usual, en general, las estadística RECMP y $U-T h e i l$ lo muestran, el desempeño se deteriora a medida que el horizonte de pronóstico incrementa ${ }^{11}$ De allí es claro que el BVAR se hubiera desempeñado mejor que la caminata aleatoria univariada (note los valores de las estadísticas U-Theil) e incluso que el VAR frecuentista.

La figura 3 ilustra los pronósticos tipo rolling de la inflación. En la misma dirección el cuadro 3 muestra los resultados de las estadísticas de DM para inflación, evaluadas para el mismo período y usando como modelo de referencia una caminata aleatoria univariada. Note que en ningún caso, empleando los niveles de significación convencionales, se logra rechazar la hipótesis nula de igualdad de bondad de pronósticos de cada modelo VAR y BVAR y el de la caminata aleatoria.

El resultado a corto plazo, 1-2 trimestres en adelante, muestra niveles adecuados, pero el pronóstico de mediano plazo, 6 a 8 trimestres, presenta demasiada incertidumbre.

Actualmente las autoridades económicas, las organizaciones internacionales y otras agencias locales acostumbran considerar los pronósticos puntuales acompañados con un indicador de incertidumbre, lo cual diferencia los modelos de otros métodos. Para ilustración, se presenta el resultado de los pronósticos en tiempo real, ex-ante, es decir, después del final de la muestra (2010-IV). La figura 4 presenta los pronósticos de inflación anual fuera de muestra desde 2011-I hasta 2012-IV con sus respectivos intervalos de pronóstico empleando para ello el modelo BVAR antes seleccionado, por ser éste el que mejores pronósticos de inflación genera. El modelo predice que la inflación anual colombiana descenderá, con pequeños repuntes, hasta alcanzar niveles de $1.3 \%$ (promedio trimestral) al final del 2012. El resultado de intervalo del pronóstico es importante cuando el banco central trabaja bajo el esquema de inflación objetivo, como lo hace el Banco de la República desde el año 2000, estos mustran aumento de la incertidumbre para finales del año 2011.

\section{Comentarios finales}

En este trabajo se estimaron modelos VAR con aplicación de las técnicas bayesianas. El pronóstico fuera de muestra de un modelo de Vectores Autorregresivos con técnicas bayesianas (BVAR), el cual contiene Precios al Consumidor, PIB, la

$\overline{10}$ No obstante, los resultados completos están disponibles para quien los requiera.

${ }^{11}$ Es de resaltar que un modelo con estadístico $U-T h e i l$ mayor a uno, no necesariamente significa un pronóstico inadecuado. 


\begin{tabular}{|c|c|c|c|c|c|c|}
\hline Modelo & $\overline{\text { EM }}$ & EAMI & $\overline{\text { EAPMP }}$ & EECPM & RECPMP & U-Theil \\
\hline & & & -promedio (1 & $, \ldots, 12)$ & & \\
\hline $\operatorname{BVAR}(\mathrm{N}, \mathrm{r}=0)$ & 0.607 & 1.197 & 27.804 & 1.584 & 35.401 & 0.727 \\
\hline NAIVE & -0.839 & 1.791 & 56.273 & 2.201 & 85.793 & 1 \\
\hline VAR (D) & -0.846 & 1.814 & 55.485 & 2.286 & 86.017 & 1.005 \\
\hline $\operatorname{BVAR}(\mathrm{D})$ & -0.638 & 1.987 & 59.096 & 2.43 & 87.317 & 1.128 \\
\hline $\operatorname{BVAR}(\mathrm{N}, \mathrm{r}=1)$ & -1.653 & 2.289 & 69.239 & 2.793 & 101.98 & 1.213 \\
\hline $\operatorname{VAR}(\mathrm{N}, \mathrm{r}=1)$ & -3.805 & 3.994 & 112.162 & 4.68 & 140.371 & 1.982 \\
\hline $\operatorname{VAR}(\mathrm{N}, \mathrm{r}=0)$ & -5.508 & 5.535 & 153.888 & 5.831 & 184.389 & 2.481 \\
\hline & & & $l=1$, NOB & $5=24$ & & \\
\hline $\operatorname{BVAR}(\mathrm{N}, \mathrm{r}=0)$ & 0.143 & 0.442 & 10.614 & 0.555 & 12.736 & 0.734 \\
\hline VAR (D) & -0.076 & 0.461 & 10.566 & 0.563 & 12.827 & 0.745 \\
\hline $\operatorname{BVAR}(\mathrm{N}, \mathrm{r}=1)$ & -0.105 & 0.465 & 10.422 & 0.565 & 12.902 & 0.748 \\
\hline $\operatorname{VAR}(\mathrm{N}, \mathrm{r}=1)$ & -0.295 & 0.552 & 13.194 & 0.663 & 15.808 & 0.877 \\
\hline NAIVE & -0.129 & 0.55 & 12.916 & 0.756 & 18.655 & 1 \\
\hline $\operatorname{VAR}(\mathrm{N}, \mathrm{r}=0)$ & -0.516 & 0.677 & 16.533 & 0.816 & 20.02 & 1.08 \\
\hline BVAR (D) & 1.132 & 1.132 & 24.548 & 1.27 & 25.961 & 1.681 \\
\hline & & & $l=2 \mathrm{NOBS}$ & $=23$ & & \\
\hline $\operatorname{BVAR}(\mathrm{N}, \mathrm{r}=0)$ & 0.306 & 0.739 & 17.23 & 0.986 & 22.117 & 0.757 \\
\hline $\operatorname{VAR}(\mathrm{D})$ & -0.156 & 0.771 & 17.867 & 1.019 & 24.344 & 0.783 \\
\hline $\operatorname{BVAR}(\mathrm{N}, \mathrm{r}=1)$ & -0.303 & 0.814 & 19.014 & 1.071 & 25.682 & 0.823 \\
\hline NAIVE & -0.278 & 0.979 & 25.438 & 1.302 & 37.595 & 1 \\
\hline BVAR (D) & 1.05 & 1.148 & 24.909 & 1.357 & 27.796 & 1.042 \\
\hline $\operatorname{VAR}(\mathrm{N}, \mathrm{r}=1)$ & -0.814 & 1.145 & 28.783 & 1.381 & 35.924 & 1.061 \\
\hline $\operatorname{VAR}(\mathrm{N}, \mathrm{r}=0)$ & -1.372 & 1.492 & 39.188 & 1.803 & 49.867 & 1.385 \\
\hline & & & $l=4 \mathrm{NOBS}$ & $=21$ & & \\
\hline $\operatorname{BVAR}(\mathrm{N}, \mathrm{r}=0)$ & 0.66 & 1.327 & 31.358 & 1.732 & 40.174 & 0.824 \\
\hline BVAR (D) & 0.722 & 1.434 & 32.73 & 1.913 & 47.099 & 0.91 \\
\hline VAR (D) & -0.513 & 1.531 & 42.081 & 2.082 & 69.935 & 0.991 \\
\hline NAIVE & -0.616 & 1.55 & 48.55 & 2.102 & 81.963 & 1 \\
\hline $\operatorname{BVAR}(\mathrm{N}, \mathrm{r}=1)$ & -1.108 & 1.799 & 48.802 & 2.317 & 76.505 & 1.102 \\
\hline $\operatorname{VAR}(\mathrm{N}, \mathrm{r}=1)$ & -2.536 & 2.86 & 81.753 & 3.396 & 107.295 & 1.615 \\
\hline $\operatorname{VAR}(\mathrm{N}, \mathrm{r}=0)$ & -4.086 & 4.086 & 116.364 & 4.579 & 149.727 & 2.178 \\
\hline & & & $l=6 \mathrm{NOBS}$ & $=19$ & & \\
\hline $\operatorname{BVAR}(\mathrm{N}, \mathrm{r}=0)$ & 0.687 & 1.35 & 31.805 & 1.774 & 41.075 & 0.701 \\
\hline NAIVE & -0.953 & 2.008 & 68.502 & 2.53 & 112.835 & 1 \\
\hline BVAR (D) & -0.987 & 2.001 & 66.534 & 2.655 & 115.191 & 1.049 \\
\hline VAR (D) & -0.84 & 2.021 & 66.293 & 2.656 & 113.626 & 1.05 \\
\hline $\operatorname{BVAR}(N, r=1)$ & -1.684 & 2.313 & 76.358 & 3.022 & 127.449 & 1.194 \\
\hline $\operatorname{VAR}(\mathrm{N}, \mathrm{r}=1)$ & -3.983 & 4.07 & 121.224 & 5.026 & 162.545 & 1.986 \\
\hline $\operatorname{VAR}(\mathrm{N}, \mathrm{r}=0)$ & -6.01 & 6.01 & 168.275 & 6.315 & 202.143 & 2.496 \\
\hline & & & $l=8 \mathrm{NOBS}$ & $=17$ & & \\
\hline $\operatorname{BVAR}(\mathrm{N}, \mathrm{r}=0)$ & 0.705 & 1.347 & 31.365 & 1.801 & 40.522 & 0.643 \\
\hline NAIVE & -1.199 & 2.369 & 76.695 & 2.803 & 114.929 & 1 \\
\hline VAR (D) & -1.2 & 2.473 & 81.387 & 3 & 126.255 & 1.07 \\
\hline BVAR (D) & -1.441 & 2.532 & 83.983 & 3.035 & 129.244 & 1.083 \\
\hline $\operatorname{BVAR}(\mathrm{N}, \mathrm{r}=1)$ & -2.099 & 2.977 & 97.285 & 3.459 & 144.698 & 1.234 \\
\hline $\operatorname{VAR}(\mathrm{N}, \mathrm{r}=1)$ & -5.311 & 5.377 & 153.415 & 6.351 & 191.194 & 2.265 \\
\hline $\operatorname{VAR}(\mathrm{N}, \mathrm{r}=0)$ & -7.346 & 7.346 & 201.536 & 7.54 & 234.56 & 2.69 \\
\hline
\end{tabular}

Fuente: Cálculos del autor

Cuadro 2. Estadísticas de Bondad de Pronóstico, Inflación 2005-I a 2010-IV.

tasa de interés y la base monetaria de la economía colombiana. Se compara el pronóstico de la inflación con el de una caminata aleatoria univariada, y contra los modelos VAR convencional de la misma especificación. Los resultados de los modelos BVAR en varias de las configuraciones mejoran en bondad estadística de pronóstico a sus rivales, al menos, en lo que refiere a la inflación.

De los pronóstico BVAR el que no restringe el pronóstico para que cumpla relaciones de cointegración, usa 6 rezagos (BVAR-5) y genera mejores pronósticos 


\begin{tabular}{|c|c|c|c|c|}
\hline Modelo & $D M_{1}$ & P-Valor $(L$ & $D M_{2}$ & $\operatorname{lor}\left(D M_{2}\right)$ \\
\hline & & Prome & $=1$ & \\
\hline $\operatorname{BVAR}(\mathrm{N}, \mathrm{r}=0)$ & 1.93 & 0.97 & 1.2 & 0.89 \\
\hline NAIVE & 2.62 & 1 & 2.92 & 1 \\
\hline VAR (D) & 3.67 & 1 & 2.93 & 1 \\
\hline BVAR (D) & 4.18 & 1 & 3.57 & 1 \\
\hline $\operatorname{BVAR}(\mathrm{N}, \mathrm{r}=1)$ & 3.82 & 1 & 3.51 & 1 \\
\hline $\operatorname{VAR}(\mathrm{N}, \mathrm{r}=1)$ & 7.89 & 1 & 7.42 & 1 \\
\hline $\operatorname{VAR}(\mathrm{N}, \mathrm{r}=0)$ & 8.59 & 1 & 8.42 & 1 \\
\hline & & $l=1$, & $\mathrm{BS}=$ & \\
\hline $\operatorname{BVAR}(\mathrm{N}, \mathrm{r}=0)$ & 2.19 & 0.99 & 2.46 & 0.99 \\
\hline VAR (D) & 3.5 & 1 & 3.53 & 1 \\
\hline $\operatorname{BVAR}(\mathrm{N}, \mathrm{r}=1)$ & 3.28 & 1 & 3.62 & 1 \\
\hline $\operatorname{VAR}(\mathrm{N}, \mathrm{r}=1)$ & 5.26 & 1 & 5.54 & 1 \\
\hline NAIVE & 4.2 & 1 & 6.49 & 1 \\
\hline $\operatorname{VAR}(\mathrm{N}, \mathrm{r}=0)$ & 4.78 & 1 & 5.29 & 1 \\
\hline BVAR (D) & 6.39 & 1 & 6.54 & 1 \\
\hline & & $l=2$ & $\mathbf{B S}=$ & \\
\hline $\operatorname{BVAR}(\mathrm{N}, \mathrm{r}=0)$ & 3.07 & 1 & 2.52 & 0.99 \\
\hline $\operatorname{VAR}(\mathrm{D})$ & 2.46 & 0.99 & 1.95 & 0.97 \\
\hline $\operatorname{BVAR}(\mathrm{N}, \mathrm{r}=1)$ & 3.74 & 1 & 3.28 & 1 \\
\hline NAIVE & 2.43 & 0.99 & 2.63 & 1 \\
\hline BVAR (D) & 6.17 & 1 & 6.2 & 1 \\
\hline $\operatorname{VAR}(\mathrm{N}, \mathrm{r}=1)$ & 4.41 & 1 & 5.08 & 1 \\
\hline $\operatorname{VAR}(\mathrm{N}, \mathrm{r}=0)$ & 5.57 & 1 & 5.44 & 1 \\
\hline
\end{tabular}

Harvey et al [1]. Fuente: Cálculos del autor

Cuadro 3. Estadísticas de Diebold-Mariano modificada. Inflación 2000-I a 2008-II.

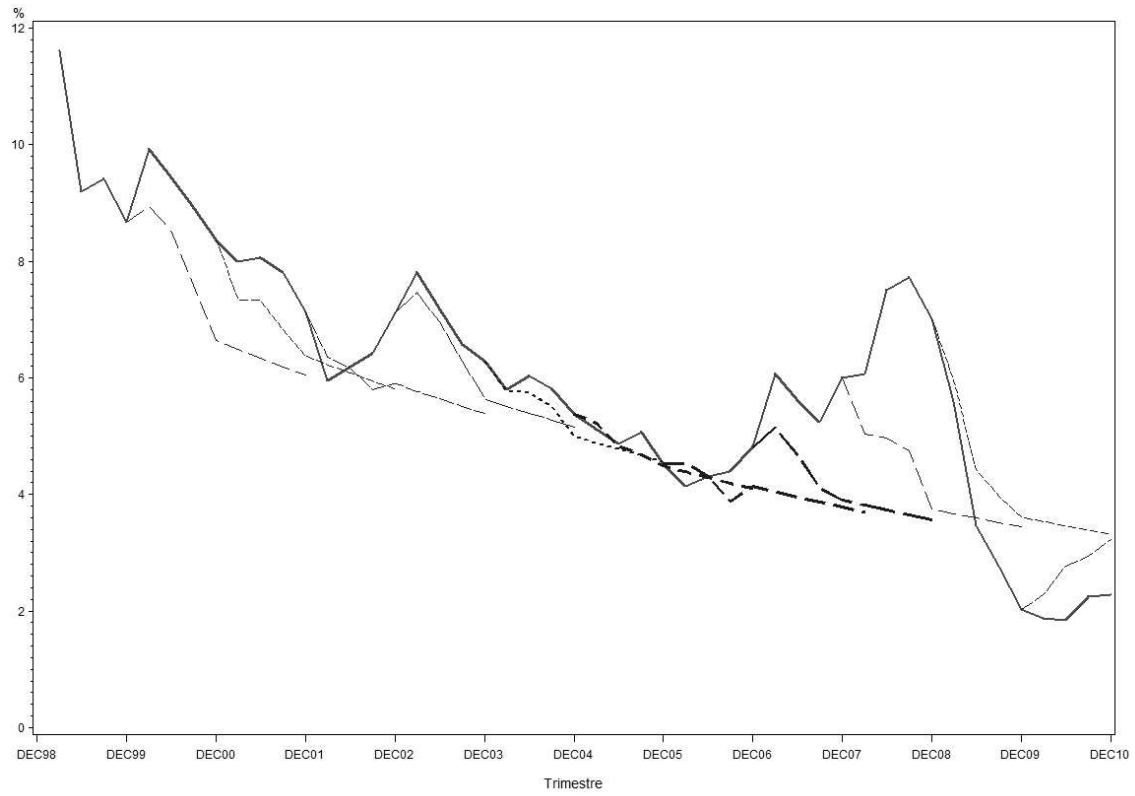

Figura 3. Pronósticos rolling de inflación. 


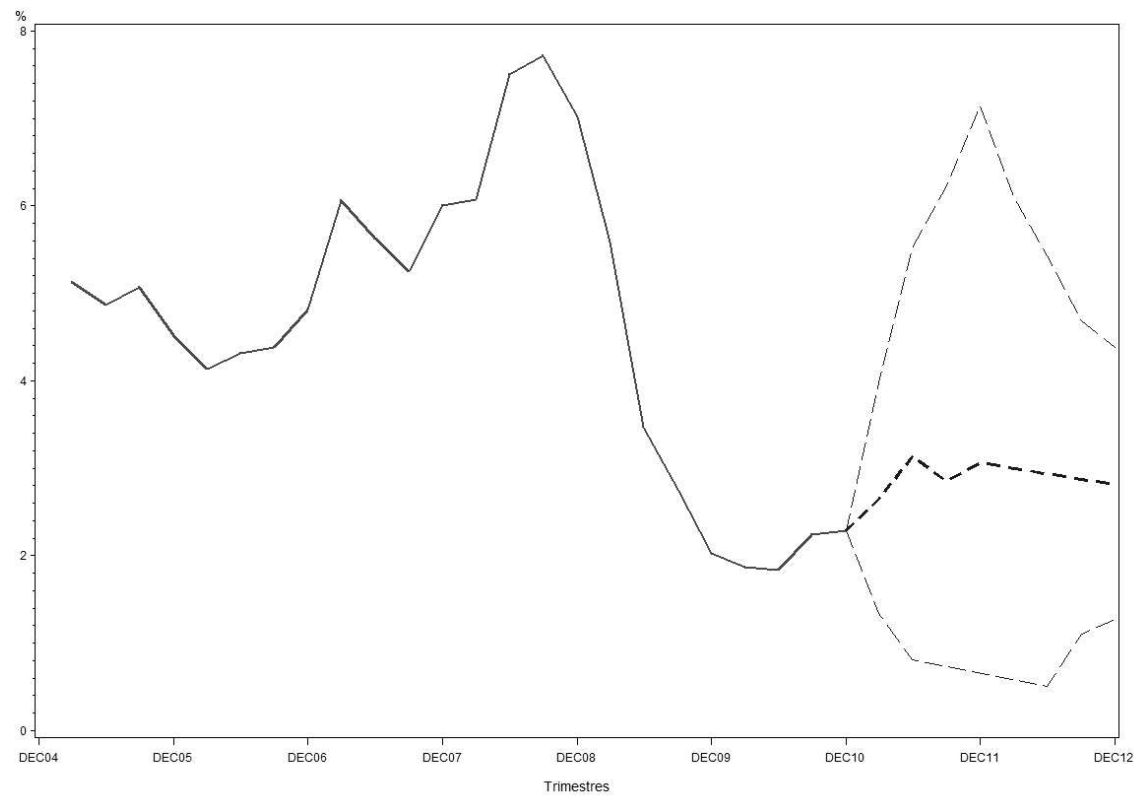

Figura 4. Inflación: Pronóstico fuera de muestra hasta 2012-IV con intervalos de credibilidad.

de la inflación para el período considerado, el término de RECPM. Reduciendo este indicador desde el $32.0 \%$ respecto al VAR, cuando se pronostica un trimestre adelante, y hasta del $76.1 \%$ cuando la predicción se hace a ocho trimestres.

Este trabajo, entonces, muestra alguna evidencia empírica relacionada con el desempeño de las posibles especificaciones VAR bayesianas para pronosticar la inflación. En consideración a que los modelos VAR están siendo cada vez mas usados para generar pronósticos, se espera que técnicas como la usada en este artículo ganen relevancia entre los usuarios tanto del sector académico como privado e institucional.

En resumen, los hallazgos aquí presentados sugieren que los BVAR que no incorporan restricciones de cointegración proporcionan una alternativa prometedora y práctica para producir pronósticos más adecuados para la inflación en Colombia. Este resultado puede servir para complementar un modelo estructural y ayudar a mejorar la bondad del pronóstico, aportando una visión alternativa a los pronósticos existentes.

No está de más advertir que éste no pretende ser un trabajo que utilice todos los resultados teóricos disponibles hoy en día ni mucho menos proponer metodologías inéditas, por el contrario, es apenas un trabajo modesto inicial que, si al caso pretende algo, es ser una referencia para futuros trabajos que usando datos de la economía colombiana apliquen técnicas más novedosas ya disponibles en la literatura internacional. En ese orden de ideas, se listan abajo algunas de 
las posibilidades para la agenda de investigación tanto para estadísticos como para econometristas y economistas.

Como trabajo de investigación futura, valdría la pena ensayar este ejercicio usando datos mensuales, o más aún frecuencias mezcladas (datos trimestrales con cifras preliminares mensuales) como en [21]. También, se puede pensar en modelos BVAR con otras priors, por ejemplo no conjugadas, lo cual conduce a la necesidad de usar métodos computacionales como los MCMC, así como alternativas de BVAR, considerando estacionalidad explícitamente y en forma dinámica como en [20] ó [19]. En un contexto un poco diferente, sería interesante intentar ajustar y usar modelos BVAR con parámetros cambiantes en el tiempo, como [1] sugiere o como lo hacen en 21] para considerar los cambios inducidos por la estrategia de inflación objetivo. Queda pendiente evaluar el efecto que ha de tener el excluir coeficientes de rezagos no significativos en cada ecuación de la representación VAR, es decir, estimar con restricciones de igualdad a cero de algunos coeficientes. En otra línea de investigación queda evaluar los pronósticos condicionales como en [23].

\section{Referencias}

1. Canova, F.: Methods for Applied Macroeconomic Research. Princeton University Press, Princeton, New Jersey. (2007)

2. Christiano, L., Eichenbaum, M., Evans C.: Nominal Rigidities and the Dynamic Effects of a Shock to Monetary Policy. Journal of Political Economy, 2005, vol. 113, No. 1, pp. 1-45. (2005)

3. Diebold, F. X., Mariano R.S.: Comparing Predictive Accuracy. Journal of Business and Economic Statistics, 13, 253-263. (1995)

4. Doan, T., Litterman R. B., Sims Ch. A.: Forecasting and Conditional Projection Using Realistic Prior Distributions. Econometric Reviews, 3, 1-100. (1984)

5. Efron, B.: Bootstrappnig Methods: Another Look at the Jacknife. Annals of Statistics, 7, 1-26. (1979)

6. Eichenbaum, M., Evans C.: Some Empirical Evidence on the Effects of Shocks to Monetary Policy on Exchange Rates. The Quarterly Journal of Economics, Vol. 110, No. 4. (Nov.), pp. 975-1009. (1995)

7. Goméz, V., Maravall, A.: Program SEATS and TRAMO: Instructions for the user. Working Paper No. 9682, Banco de España. (1996)

8. González A., Melo, L. F., Posada C. E.: Inflación y Dinero en Colombia: Otro Modelo P-Estrella. Borrador de Economía 418, Banco de la República, noviembre. (2006)

9. González E., Grajales A. : Pronósticos directos de la inflación colombiana. Borrador de Economía 458, Banco de la República, octubre. (2007)

10. González E., Melo L. F., Monroy V., Rojas B.: A Dynamic Factor Model for the Colombian Inflation. Borrador 549 Banco de la República, enero. (2009)

11. Harvey, D. I., Leybourne S. J., Newbold P.: Testing the Equality of Prediction Mean Squares Errors. International Journal of Forecasting, vol. 13, pp 281-291. (1997)

12. Johansen, S.: Likelihood-based Inference in Cointegrated Vector Autoregresive Models, Oxford University Press, Oxford. (1995) 
13. Judge, G. G., Griffiths, W. E., Hill, R. C., Lütkepohl H., Lee T-C.: Introduction to the Theory and Practice of Econometrics, Second Edtion, John Wiley, New York. (1989)

14. Koop, G., Strachan R., Van Dijk H., Villani M.: Bayesian Approaches to Cointegration, in K. Patterson and T. C. Mills (eds), Palgrave Handbook of Econometrics, Volume 1: Econometric Theory, Palgrave Macmillan, Houndmills. (2005)

15. Langebaek, A., González E.: Inflación y precios relativos en Colombia. Borradores de Economía, 459 Banco de la República. (2007)

16. Litterman, R. B.: A Bayesian Procedure for Forecasting with Vector Autorregresions. Federal Reserve Bank of Minneapolis, Working Paper. (1981)

17. Litterman, R. B.: Forecasting with Bayesian Vector Autoregressions - Five Years of Experience. Journal of Business and Economic Statistics, vol. 4, No 1, pp. 25-38. (1986)

18. Lütkepohl, H.: New Introduction to Multiple time Series Analysis. Springer, Berlin. (2005)

19. Quillis, E. M.: Modelos BVAR: Especificación, Estimación e Inferencia, WP: Papeles de Trabajo Editados por el Instituto de Estudios Fiscales, No. 8, España. (2002)

20. Raynauld, J., Simonato J. G.: Seasonal BVAR Models, Journal of Econometrics, vol. 55, pp. 203-229. (1993)

21. Robertson, J. C., Tallman, E. W.: Vector Autoregressions: Forecasting and Reality. Federal Reserve Bank of Atlanta, Economic Review, first quarter, páginas 4-18. (1999)

22. Sims, Ch. A.: Macroeconomics and Reality, Econometrica, vol. 48, No. 1, pp. 1-48. (1980)

23. Villani, M.: Inference in Vector Autoregressive Models with an Informative Prior on the Steady State. Sveriges Riksbank Working Paper Series, No. 181, March, Stockholm. (2005) 